\title{
Joint Color Quantization and Dithering Techniques
}

\author{
Mohammed Ahmed Hassan \\ Assistant Professor, Department of Applied and Computer Sciences \\ Seiyun Community College \\ Seiyun, Yemen
}

\begin{abstract}
Color quantization process is considered in two stages: the selection of an optimal color palette and the mapping of each pixel of the image to a color from the color palette. Since the color palette is limited, some disturbing degradations such as false contours are visible on delivered color quantized images. A common way to overcome this problem is the use of dithering techniques. In this paper, two methods for color quantization are proposed for the use with color dithering techniques in a way that better results will be obtained after dithering. The results show that the proposed methods, when used with dithering techniques, significantly improve the visual quality of the resulting color quantized images compared to the traditional color quantization algorithms.
\end{abstract}

\section{General Terms}

Color Quantization, Dithering Techniques.

\section{Keywords}

Color Images, fractal dimensions, error diffusion, combined quantization and error diffusion.

\section{INTRODUCTION}

Color quantization is the process of reducing the number of colors presented in a digital full color image. Originally, color quantization has been used to satisfy the display hardware constraints that allow only a limited number of colors to be displayed simultaneously. Today the original motivation of color quantization has changed due to the availability of inexpensive full color displays. However, color quantization is still an important problem in the fields of image processing and computer graphics [2]. It can be used in mobile and hand-held devices where memory is usually small [26], it can be used for low-cost display and printing devices where only a small number of colors can be displayed or printed simultaneously [29]. It also has been used as a preprocessing step for many applications such as object recognition [31], image compression [35], and content-based image retrieval (CBIR) [32].

Regardless of color quantization algorithm used, reconstruction of an image with a limited number of representative colors (color palette) will cause highly visible degradations in image quality. The most disturbing of these degradations is the appearance of false contours. False contours appear when batches of colors in a smooth gradient area are mapped into a constant palette colors forming a flat regions; boundaries between such regions may be visible as false contours as shown in Fig. 1

A common way to overcome the problem of false contours is the use of dithering techniques. Dithering techniques make use of the averaging property of the human eye to the colors in a neighborhood of the point of interest and create the illusion of more colors. A dithering technique called error diffusion achieves this effect by distributing the error encountered in quantizing a pixel to the neighboring pixels. This results in an alternation of palette colors in the neighborhood and is perceived as a new color by the

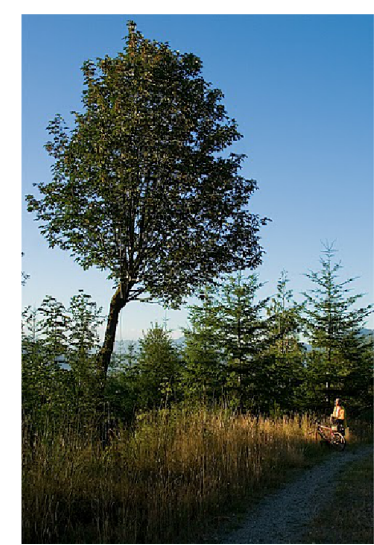

(a)

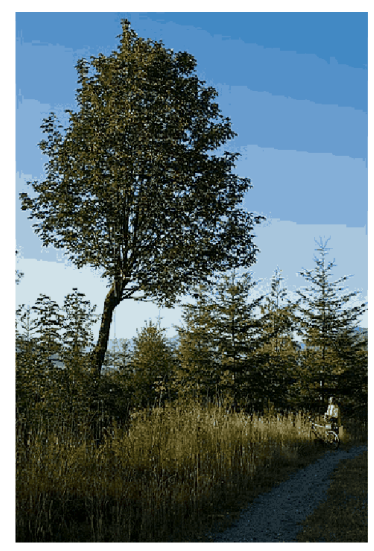

(c)

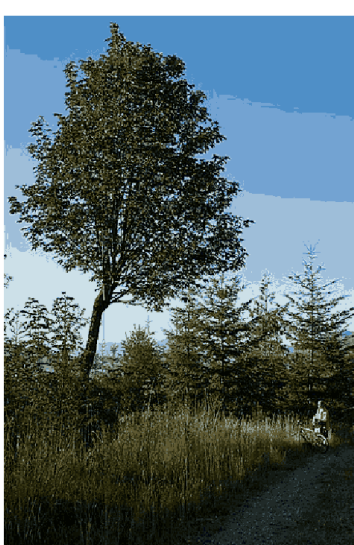

(b)

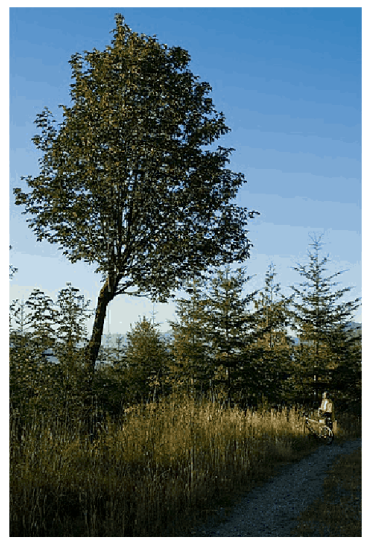

(d)
Fig. 1: Appearance of false contours in color quantized images. (a) Full color image, (b) Color quantized image with 16 colors, (c) Color quantized image with 32 colors, (d) Color quantized image with 64 colors.

eye. Some well-known error diffusion filters are Floyd-Steinberg [4], Stucki [30], and Jarvice-Judice-Ninke [9].

Usually color quantization and dithering are performed sequentially; the quality of the dithering process depending on the selection of the color palette. Standard methods of color quantization do not take dithering into account. Therefore, the desirable qualities obtained by quantization are often disturbed by the dithering process. The most commonly used algorithms for color quantization are: median-cut [8], center-cut [10], octree [7], k-means [15], Wu's quantizer [34], fuzzy C-means [12], and SOM [3].

There is some previous work for jointly quantizing and dithering color images in the literature. Orchard [19] used binary tree splitting (BTS) to create the color palette that minimizes the total squared difference between the actual and the quantized images and then modified dithering techniques are combined with quantization process. A modified BTS quantizer of [19] is proposed 
in [1] in such a way that at leaves the pair of cluster centers are given equal displacements from each other following the split in order to span a larger volume of color space. This creates an illusion of more colors after dithering. In competitive learning and error diffusion algorithm [29], the quantization error is diffused to neighboring pixels during learning process. In [21] three new fuzzy methods to jointly quantize and dither the color images are presented; in two of them a fraction of the quantization error is distributed to neighboring pixels during creating color palettes and then perform quantization and dithering as usual with the resultant palette, while the third method is to enlarge the convex hull of the quantization colors to obtain a color palette which is more suitable for dithering.

This paper is organized as follows: Section 2 explains the Optimal Color Palette for Error Diffusion Techniques method. In Section 3, the Local Fractal Dimension-Based Color Quantization method is presented. This study is concluded in Section 4.

\section{OPTIMAL COLOR PALETTE FOR ERROR DIFFUSION TECHNIQUES}

In this section, the first method for quantization of color images to be used with error diffusion techniques is presented. It consists of three steps: in the first step, the colors in original image are quantized into a number of colors that is much smaller than the desired number of colors. In the second step, the remaining palette colors are selected around each palette color generated in the first step based on the CIELAB color space Just Noticeable Color Difference (JNCD) threshold, and also on the size of the clusters. Finally, error diffusion technique is incorporated within the process of pixel mapping.

\subsection{Choice of Color Space}

The CIE $L^{*} a^{*} b^{*}$ (CIELAB) and $L^{*} u^{*} v^{*}$ (CIELUV) color spaces [25] are considered perceptually uniform and referred to as uniform color spaces in which the Euclidean distances between different colors in the color space correspond approximately to perceived color differences. The application of uniform color spaces to the problem of color quantization has been studied by Gentile et al. [5] and to half-toning [6], the results indicate that such color spaces can significantly improve quality of the quantized image relative to the standard RGB color space. The CIE recommended using XYZ coordinate system to transform RGB to $L^{*} a^{*} b^{*}$. The following equations are used to transfer RGB to XYZ.

$$
\left(\begin{array}{l}
X \\
Y \\
Z
\end{array}\right)=\left(\begin{array}{lll}
0.4124564 & 0.3575761 & 0.1804375 \\
0.2126729 & 0.7151522 & 0.0721750 \\
0.0193339 & 0.1191920 & 0.9503041
\end{array}\right)\left(\begin{array}{l}
R \\
G \\
B
\end{array}\right)
$$

And then from XYZ to CIELAB color space

$$
\begin{gathered}
L^{*}= \begin{cases}116\left(Y / Y_{n}\right)^{1 / 3}-16, & \text { if } Y / Y_{n}>0.008856 \\
903.3\left(Y / Y_{n}\right), & \text { if } Y / Y_{n} \leq 0.008856\end{cases} \\
a^{*}=500\left(f\left(X / X_{n}\right)-f\left(Y / Y_{n}\right)\right) \\
b^{*}=200\left(f\left(Y / Y_{n}\right)-f\left(Z / Z_{n}\right)\right)
\end{gathered}
$$

where

$$
f(t)= \begin{cases}t^{1 / 3}, & \text { for } t>0.008856 \\ 7.787 * t+16 / 116, & \text { for } t \leq 0.008856\end{cases}
$$

Here $Y_{n}=1.0$ is the luminance, and $X_{n}=0.950455, Z_{n}=$ 1.088753 are the chrominances for the $D_{65}$ white point.

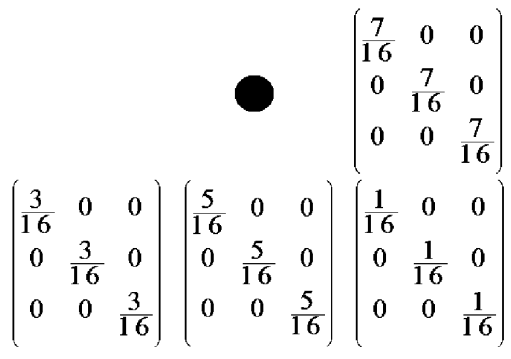

Fig. 2: Floyd-Steinberg vector filter for a single pixel.

Two colors in CIELAB color space are perceptually distinguishable from each other if the Euclidean distance between these two colors is greater than a threshold value $3[16]$. This threshold is known as the Just Noticeable Color Difference (JNCD) threshold.

\subsection{The Proposed Color Quantization Method}

After transforming the input image into the CIELAB color space, the quantization process is composed of the following steps:

\section{-Step 1: Generation of Initial Palette Colors}

In this step, the colors in the original image are quantized into a small set of $M$ colors that is much less than the desired $K$ palette colors $\left(M=2^{\left\lfloor\frac{\log _{2} K}{2}\right\rfloor}\right)$ using k-means algorithm or any other quantization algorithm.

\section{-Step 2: Selection of the Remaining Palette Colors}

After the generation of the initial palette colors, the remaining $(K-M)$ palette colors are selected around the initial palette color based on CIELAB JNCD threshold value 3 . The use of CIELAB JNCD threshold is to ensure that the selected palette colors are far from each other by a distance that is greater than the CIELAB JNCD threshold to be distinguishable from each other. The number of selected palette colors around a given initial palette color is proportional to the size of cluster of image colors represented by that initial palette color.

For an initial palette color $P_{i}$ that represents a cluster $\left\{C_{1}, C_{2}, \ldots, C_{R_{i}}\right\}$ of image colors, a set $S_{i}$ of $\frac{R_{i}}{N}(K-$ $M)$ distinct image colors will be selected around $\stackrel{N}{P}_{i}$ from $\left\{C_{1}, C_{2}, \ldots, C_{R_{i}}\right\}$ and are assigned as new palette colors. $N$ is the total number of distinct colors in the original image. The selection of the $S_{i}$ palette colors around $P_{i}$ is done as follows:

$P_{i_{j}}=\min _{P \in\left\{P_{i_{1}}, P_{i_{2}}, . ., P_{i_{j-1}}\right\}}\left\{d(P, C)>3: C \in\left\{C_{1}, C_{2}, . ., C_{R_{i}}\right\}\right\}$

$$
j=2, \ldots,\left|S_{i}\right|+1,\left(P_{i_{1}}=P_{i}\right)
$$

where $d$ is the Euclidian distance between two colors in the CIELAB color space.

\section{-Step 3: Pixel Mapping with Error Diffusion}

Once the color palette has been designed, the quantized image is produced by mapping each pixel to the closest color from the color palette. To overcome the problem of false contours that are commonly sensed in color quantized images, error diffusion technique is incorporated within the pixel mapping process. The pixels in the original image are chosen in raster ordering from left to right and top to bottom. Each pixel is quantized and the quantization error is propagated forward to the neighboring pixels that have not yet been quantized. Fig. 2 shows Floyd-Steinberg [4] filter that is used to perform the error diffusion. 


\subsection{Results and Discussions}

Standard color quantization process is generally considered as a clustering problem to find the $K$ palette colors that minimize some error criterion for all the colors in an image. However, two problems are associated with performing error diffusion techniques for color quantized images. The first problem is, if a given color is close to the boundary of a color cluster, the quantization errors accumulate and eventually a palette color from a different cluster is produced. This exposes itself as a color impulse that is very disturbing. On the other hand, in smooth regions usually the colors are very close to a palette color with a small dithered error. Therefore those colors will be mapped to the same palette color forming flat regions. This results the existence of some false contours after dithering. These two problems disappeared in the proposed method because the palette colors are distributed around few initially selected palette colors. Therefore it needs a small diffused error to jump from one palette color into another close palette color in the same cluster. This results a high alternation of palette colors between neighboring pixels to give full illusion of smooth gradation and hence reduces visible false contours. The selection of palette colors from the same cluster will avoid the presence of color impulses.

In Fig. 3 and Fig. 4 two full color images are quantized by the proposed method. The same two images are also presented to four popular quantization algorithms namely: k-means [13], median-cut [8], octree algorithm [7], and SOM [3], in which the Floyd-Steinberg error diffusion was incorporated within the pixel mapping process. It is observed that applying the FloydSteinberg dithering on the other quantization algorithms causes the creation of color impulses. The color impulses are in the form of isolated green and dark blue pixels on sky area in Fig. 4(b)(f). It is also observed that some false contours remain visible in these images in the smooth gradient area in Fig. 3. b)-(f). Fig. 3 a) and Fig. 4 a) are the results of the proposed method that shows better performance when compared to the other algorithms. As expected, disturbing colored impulses and false contours are minimized compared with other algorithms.

\section{LOCAL FRACTAL DIMENSION-BASED COLOR QUANTIZATION FOR ERROR DIFFUSION TECHNIQUES}

In this section, the second method for color quantization to be used with dithering techniques is presented. The premise of the proposed method is to improve the perceived quality in low activity (smooth) regions where false contours in an image are most likely to occur while preserving structure that is commonly lost in high activity (busy) regions when applying dithering techniques. Improving the perceived quality in low activity regions is achieved by introducing more colors to regions with low activity in order to move quantization errors from low activity regions to high activity regions. The errors will be less visible to the human eye as the human visual system is less sensitive to quantization errors in high activity regions than to errors in low activity regions. Preserving structure in high activity regions will be achieved by applying dithering techniques only to low activity regions where false contours in an image are most likely to occur.

\subsection{Local Fractal Dimension}

Fractal dimension of imaged 3D surfaces can be used as a measure to perceptually distinguish between smooth and rough textured regions [22 23]. It has been widely applied to many fields of digital image processing, such as texture classification and segmentation [14, 24, 11, 18], image data compression [17], and computer graphics [36].
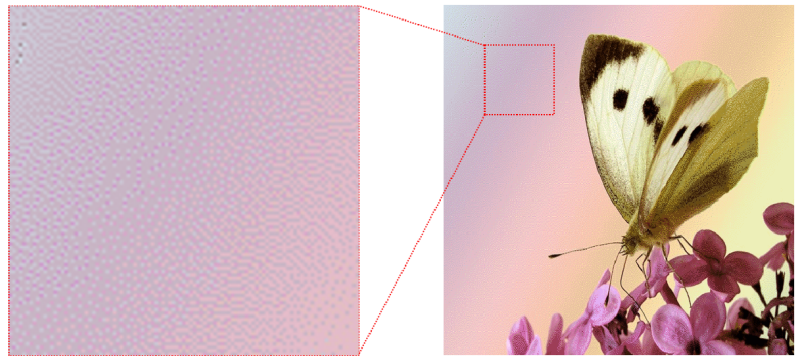

(a)
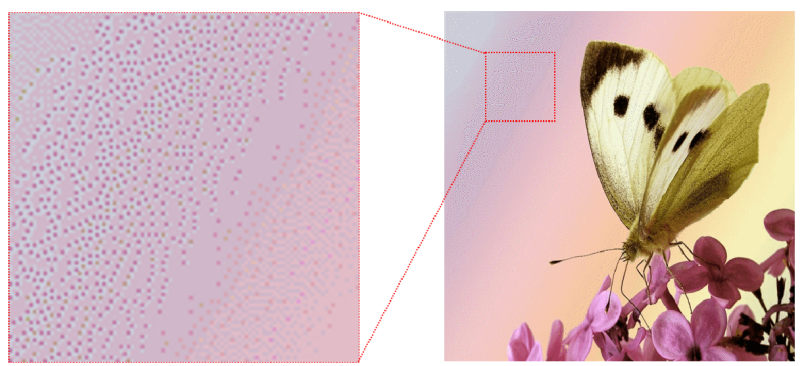

(b)
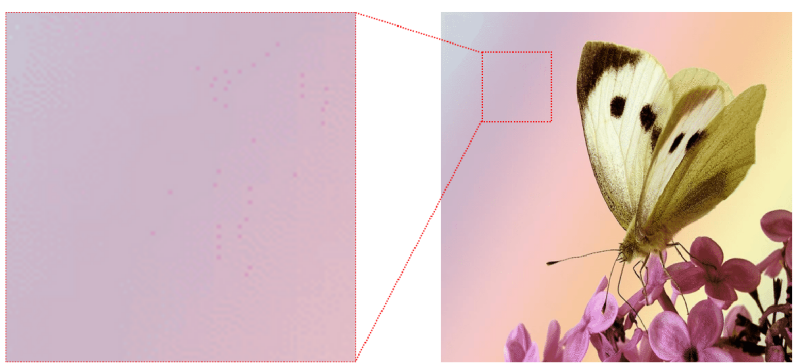

(c)
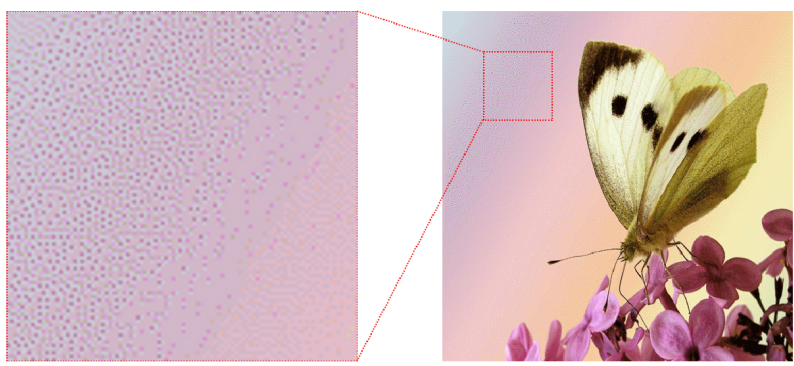

(d)
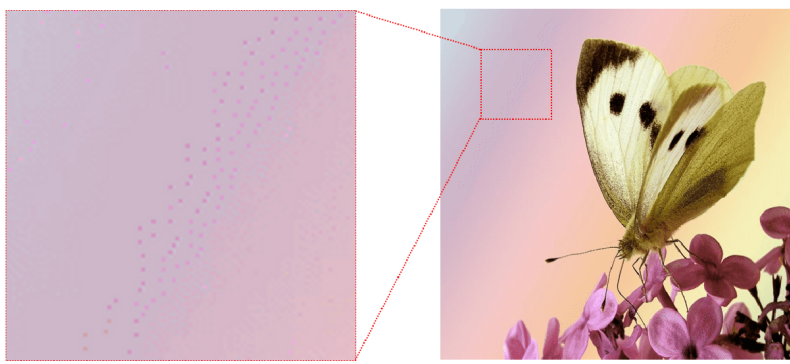

(e)

Fig. 3: Color quantized images with 128 colors followed by FloydSteinberg error diffusion. (a) Proposed method, (b) K-means, (c) Median Cut, (d) Octree, (e) SOM. 

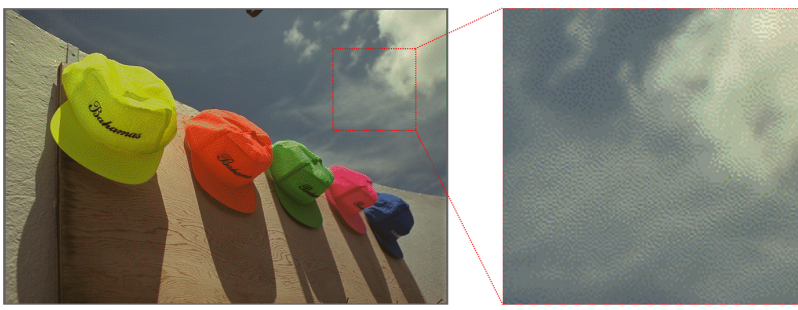

(a)
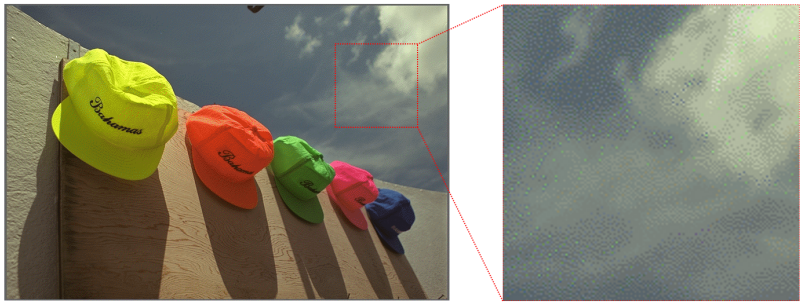

(b)
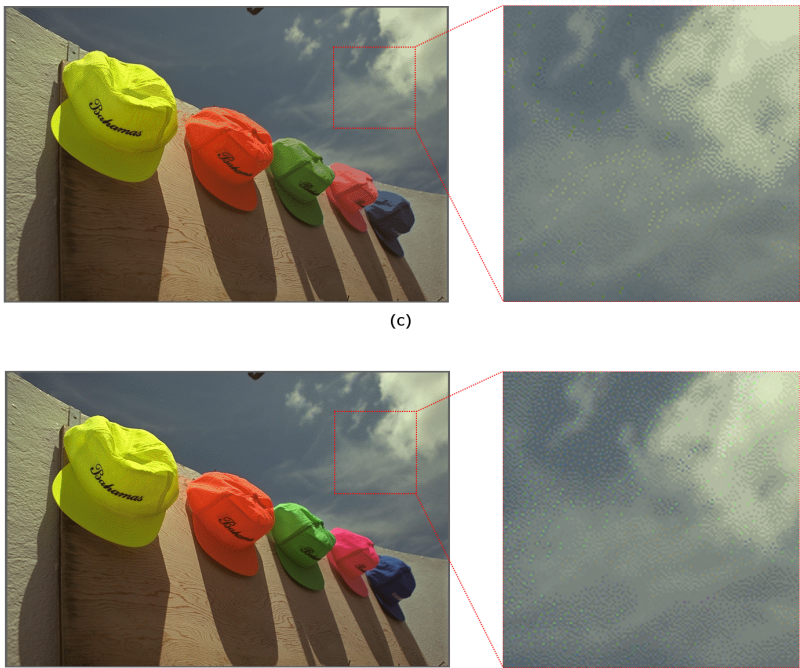

(d)
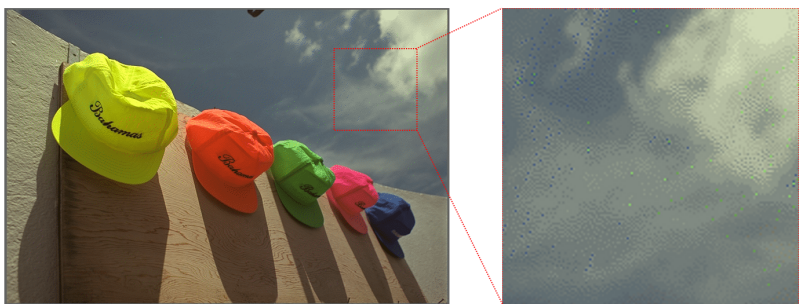

(e)

Fig. 4: Color quantized images with 128 colors followed by FloydSteinberg error diffusion. (a) Proposed method, (b) Kmeans, (c) Median Cut, (d) Octree, (e) SOM.

Sarkar and Chaudhuri [27, 28] proposed a method to find the fractal dimension of images known as differential box counting method. In their method, for an image of size $M \times M$ pixels scaled down to a size $s \times s$ where $M / 2 \geq s>1$ and $s$ is an integer. The entire image can be considered as a 3-D space with $(x, y)$ denoting 2-D position and the third coordinate $(z)$ denoting gray level. The $(x, y)$ space is partitioned into grids of size $s \times s$. On each grid there is a column of boxes of size $s \times s \times s^{\prime}$ (see Fig. 5). If the total number of gray levels is $G$, then $\left\lfloor G / s^{\prime}\right\rfloor=\lfloor M / s\rfloor$. The contribution of the $(i, j)^{t h}$ grid is

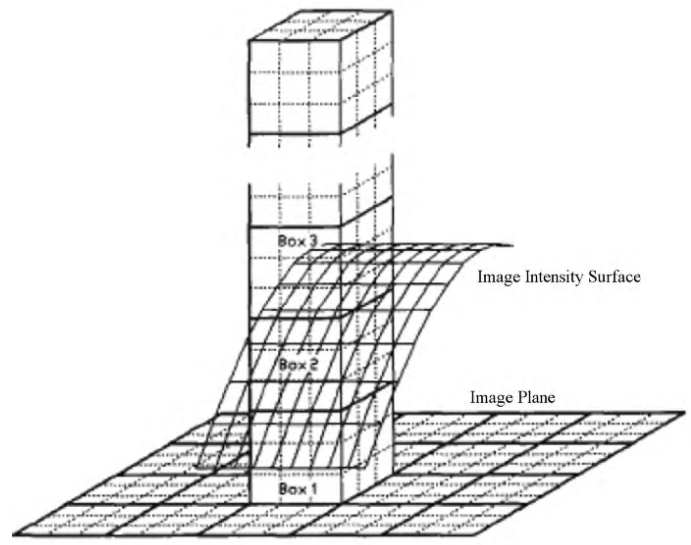

Fig. 5: Estimation of fractal dimension 28

computed as

$$
n_{r}=l-k+1
$$

where the minimum and maximum gray level of the image in the $(i, j)^{t h}$ grid fall in box number $k$ and $l$, respectively. For example in Fig. 5 where $s=s^{\prime}=3$, assign numbers 1, 2, . . to the boxes as shown. Let the minimum and maximum gray level of the image in the $(i, j)^{t h}$ grid fall in box number 1 and 3 , respectively. The contribution of the $(i, j)^{t h}$ grid is given as $n_{r}(i, j)=3-1+1$. Taking the contribution of all grids, $N_{r}=$ $\sum_{i, j} n_{r} . N_{r}$ is counted for different values of $r$, i.e., different values of $s$. Using the following equation:

$$
1=N_{r} \times r^{D} \quad \text { or } \quad D=\log \left(N_{r}\right) / \log (1 / r)
$$

The fractal dimension $D$ can be estimated from the least square linear fit of $\log \left(N_{r}\right)$ against $\log (1 / r)$.

Fig. 6 shows the original images (left) and their local fractal dimension (LFD) maps (right) where every position $(i, j)$ in the LFD map represents the LFD value estimated using a $3 \times 3$ local window centered at position $(i, j)$ in the original image. The LFD values are scaled into the rage 0-255 for display purpose. Notice how the low activity (smooth) regions are given small LFD values (darker) while the high activity (busy) regions are given large LFD values (brighter). It would be easy to distinguish between such regions and weight the importance of a pixel based on the spatial activity of the area of the image where the pixel appears. The pixel's importance (activity weighting) is inversely proportional to the activity level of the region where it appears in the image.

\subsection{Construction of Color Palette}

To describe the proposed algorithm for color image quantization using local fractal dimension (LFD) maps, the k-means [13] algorithm which is the basis of the proposed algorithm is first explained. Generally the k-means algorithm seeks to minimize an objective function which is defined as

$$
j=\sum_{i=1}^{k} \sum_{C \in S_{i}} W(C)\left\|C-\bar{C}_{S_{i}}\right\|^{2}
$$

where $\bar{C}_{S_{i}}$ is the center of the cluster $S_{i}, k$ is the number of clusters, and $W(C)$ is the weight factor of the pixel $C$. Initially the $\mathrm{k}$-means algorithm starts with a set of $k$ centers that are randomly selected. At each iterative step, scan through all the pixels of the original image and assign each pixel to the nearest center in the 

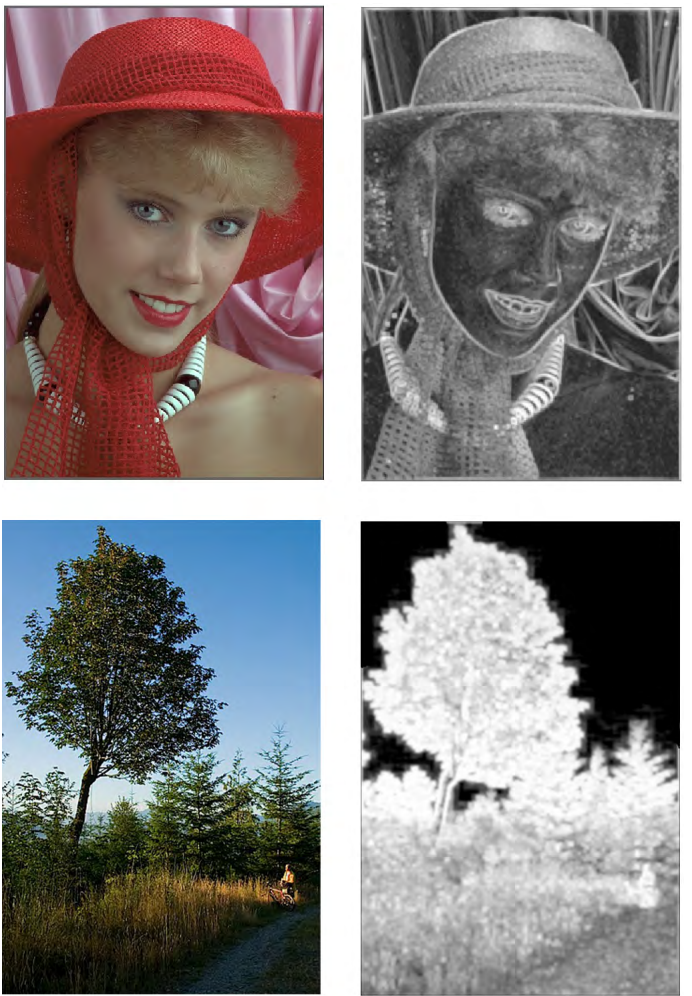

Fig. 6: LFD map of an image: (left) the original images and (right) their LFD maps

sense of minimizing 9 . Then the new centers are calculated as

$$
\bar{C}_{S_{i}}=\frac{\sum_{C \in S_{i}} W(C) \times C}{\sum_{C \in S_{i}} W(C)} \quad i=1,2, . ., k
$$

The steps are repeated until the algorithm converges or the number of iterations reaches a specified value. Usually in the conventional k-means algorithm the weight factor $W(C)=1$ for all pixels, therefore all pixels are treated equally regardless of their spatial distribution.

The proposed color quantization algorithm follows the same steps as the k-means algorithm except that the weight factor $W(C)$ of pixel $C$ at the $(i, j)^{t h}$ position in the original image is equal to the inverse of its corresponding LFD value at the $(i, j)^{t h}$ position in the LFD map. In this way pixels are not equally treated where pixels in the low activity (smooth) regions are given larger weight (importance) than those pixels in the high activity (busy) regions. Accordingly, more levels of colors will be presented in low activity regions and this will not degrade the quality of the image as the human eyes are less sensitive to quantization errors in high activity regions than in low activity regions. As a result, the perceived quality in low activity regions will be improved after dithering techniques are applied as shown in the next section. Fig. 7 shows the color quantization outputs for the conventional k-means and the proposed algorithm for 16 and 32 colors. It is clear that the proposed algorithm allocates more levels of colors to the gradient sky region than the conventional k-means does to the same region for both 16 and 32 colors.

\subsection{Pixel Mapping with Error Diffusion}

To succeed in facing up to the problem of false contours in the color quantized images, error diffusion technique within the pixel mapping process is incorporated. In conventional systems

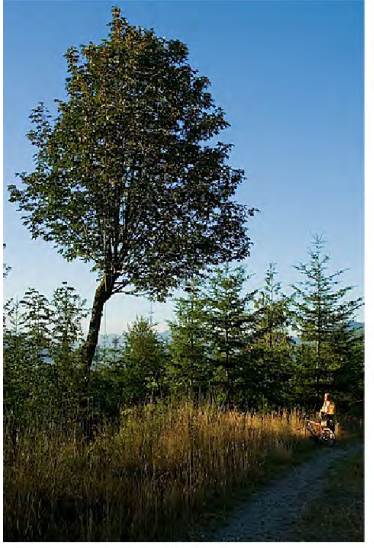

(a)

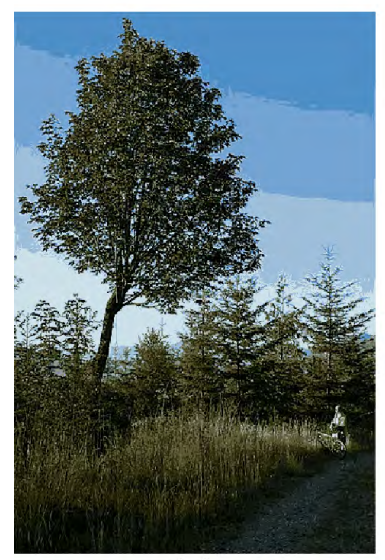

(c)

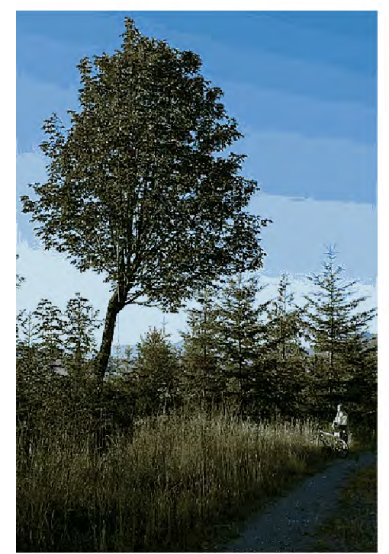

(e)

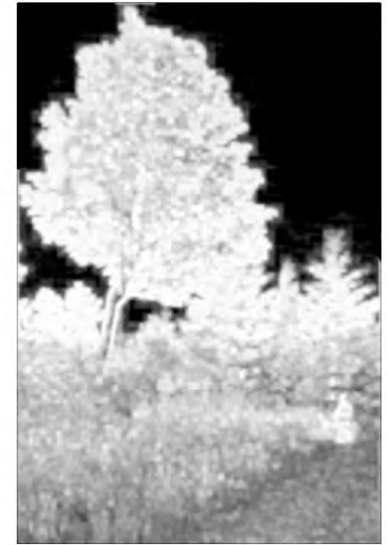

(b)

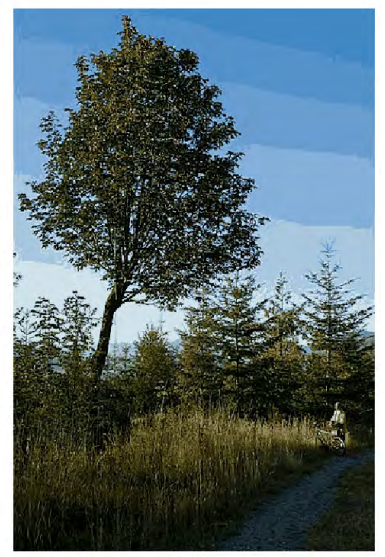

(d)

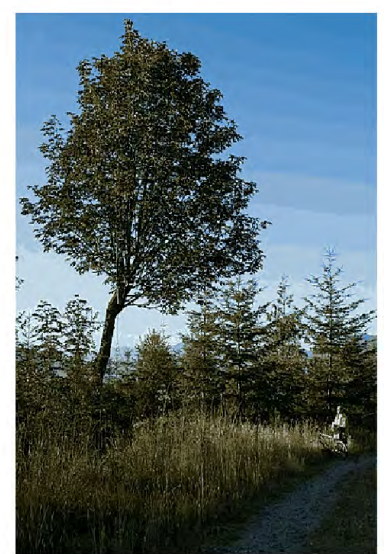

(f)
Fig. 7: Color quantization results of 'Tree' image. (a) The original image, (b) LFD map of (a), (c) K-means 16 colors, (d) k-means 32 colors, (e) Proposed method 16 colors, (f) Proposed method 32 colors.

for error diffusion, the pixels are chosen in raster ordering from left to right and top to bottom. Each pixel is quantized and the quantization error is propagated forward to the neighboring pixels that have not yet been quantized with a causal filter. One problem is associated with this approach that the structure in high activity regions may be distorted due to the accumulated quantization error (see Fig. 8(a)). To avoid unnecessarily dithering of high activity regions, the pixels in high activity regions are masked using smooth-region map in a way that the Floyd-Steinberg error diffusion filter [4] is applied only to pixels in low activity regions where false contours in an image are most likely to occur. In this 

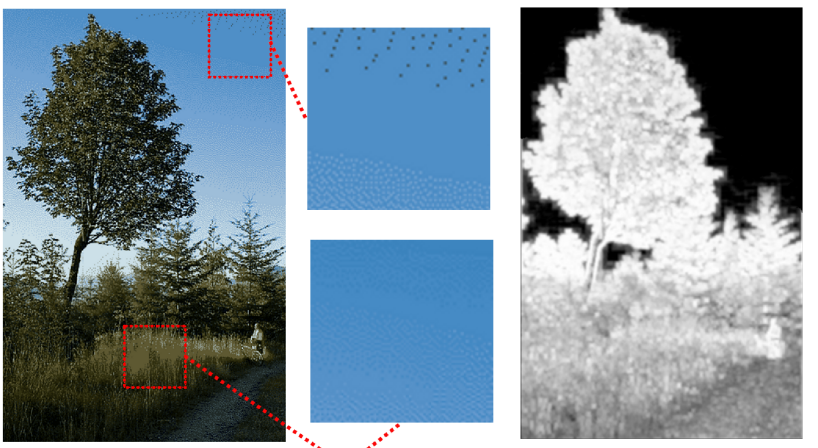

(b)
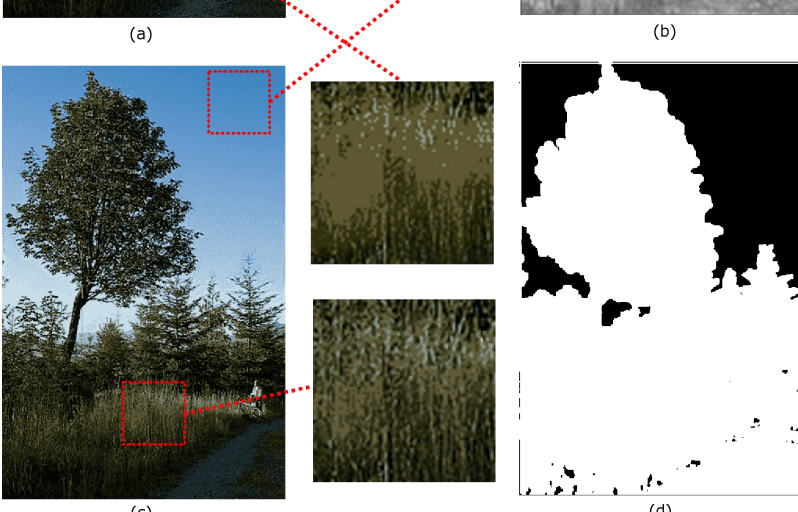

Fig. 8: Dithered quantization results of 'Tree' image. (a) k-means $16 \mathrm{col}$ ors, (b) LFD map of original image 'Tree' image, (c) Proposed algorithm 16 colors, (d) flat-region map of original 'Tree' image (high activity regions in white color and low activity regions in black color).

way false contours in low activity regions will be removed while preserving the structure in high activity regions that is commonly lost in high activity regions when applying dithering techniques. The smooth-region map is created by thresholding the LFD map using the Otsu's global image threshold method [20]. Fig. 8(b) and (d) show the LFD map of the 'Tree' image and its corresponding smooth-region map respectively.

\subsection{Results and Discussions}

In Fig. 8 (a) the full color image 'Tree' has been quantized to 16 colors using the conventional k-means where the FloydSteinberg error diffusion [4] has been incorporated within the pixel mapping process. It is observed that the application of the Floyd-Steinberg error diffusion causes the creation of color impulses and some false contours remain visible in the sky region as well as distorting structure in grass region. Those color impulses and false contours are the result of insufficient color levels presented in the gradient sky region, while the distorted structure is the result of unnecessarily dithering high activity regions where false contours in an image are not apparent to the human eye. On the other hand Fig. 8(c) shows the proposed color quantization outputs of 16 colors. It is clear that the false contours are less visible in the sky region compared to the conventional k-means and the result is free from color impulses. This is due to introducing more color levels in gradient sky region than the conventional k-means (see Fig. 7 (c) and (e)). As a result, dithering in low activity regions makes a pixel jump to another close palette color for even small errors. This results a high alternation of palette colors between neighboring pixels to give full illusion of smooth gradation. At the same time, it may also be noted that structure in the grass region is preserved by not performing dithering in the high activity regions.

For the objective evaluation of the proposed method, two image quality metrics have been used namely: the widely used peak

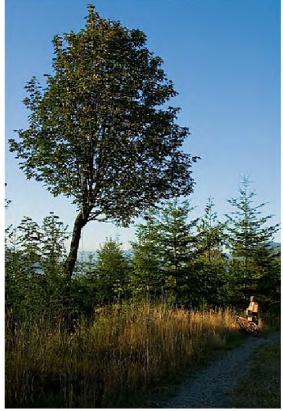

(a)

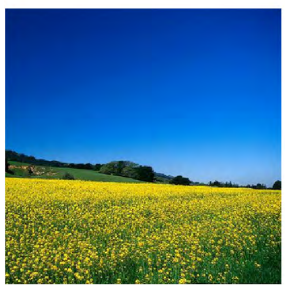

(c)

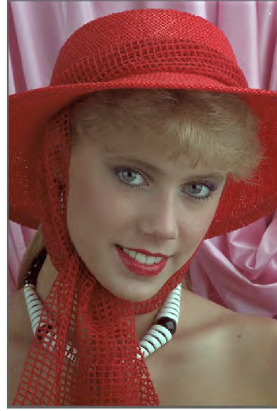

(b)

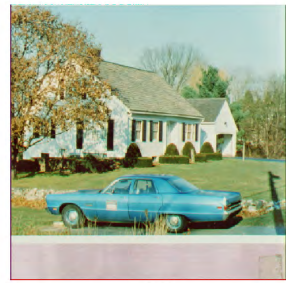

(d)
Fig. 9: Original images used for the different experiments. (a) Tree (59974 colors), (b) Woman (31744 colors), (c) Flowers (47946 colors), (d) House (154605 colors).

signal-to-noise ratio metric (PSNR) where a higher PSNR value means that the distorted image is closer to the original, and the structural similarity measure (SSIM) [33] to measure how close is the structure of the resultant dithered quantized image to the structure of the original image. Table 1 shows the performance comparison of joint color quantization and dithering for the algorithms: median-cut [8], k-means algorithm [13], and the proposed method in terms of PSNR, and SSIM metrics for the testing images in Fig. 9 The results show that the proposed method produces quantized images that are perceptually closer to the original images, and at the same time it preserves more structure of the original images when compared with the other methods.

\section{CONCLUSION}

Color quantization is an important problem for many applications in graphics and multimedia where only a limited number of colors can be displayed or printed simultaneously. In this paper, two methods for color quantization are proposed to obtain better results with minimum visual degradation after dithering techniques are applied. Simulation results show that the proposed methods give a remarkable improvement in the quality of joint color quantization and dithering both subjectively and objectively compared to the traditional quantization methods.

\section{REFERENCES}

[1] L. Akarun, D. Ozdemir, and O. Yalcin. A modified quantization algorithm for dithering of color images. Electronics Letters, 32:1185-1186, 1996.

[2] J. Braquelaire and L. Brun. Comparison and optimization of methods of color image quantization. IEEE Transactions on Image Processing, 6(7):1048-1052, 1997.

[3] A. Dekker. Kohonen neural networks for optimal colour quantization. Network Computation in Neural Systems, 5(3):351-367, 1994.

[4] R. Floyd and L. Steinberg. An adaptive algorithm for spatial grey scale. In Proceedings of the Society of Information Display, volume 17, pages 75-77, 1976. 
Table 1. : Objective validation of the LFD-based method. The validation criteria are: the widely used peak signal-to-noise ratio metric (PSNR) and the structural similarity measure (SSIM) [33].

\begin{tabular}{cc|cc|cc|cc}
\hline \hline \multirow{2}{*}{ Image } & \multirow{2}{*}{ Colors } & \multicolumn{2}{|c|}{ Median Cut+ED } & \multicolumn{2}{c|}{ K-means+ED } & \multicolumn{2}{c}{ Proposed Method } \\
\cline { 3 - 8 } & & PSNR & SSIM & PSNR & SSIM & PSNR & SSIM \\
\hline \multirow{4}{*}{ Tree } & 32 & 26.414 & 0.887 & 29.854 & 0.883 & $\mathbf{3 1 . 6 6 6}$ & $\mathbf{0 . 8 9 1}$ \\
& 64 & 28.468 & 0.914 & 32.014 & 0.924 & $\mathbf{3 5 . 2 1 4}$ & $\mathbf{0 . 9 3 4}$ \\
& 128 & 31.736 & 0.951 & 34.910 & 0.954 & $\mathbf{3 7 . 5 0 9}$ & $\mathbf{0 . 9 5 5}$ \\
& 256 & 34.287 & 0.969 & 36.924 & 0.968 & $\mathbf{3 9 . 1 2 9}$ & $\mathbf{0 . 9 7 5}$ \\
\hline \multirow{4}{*}{ Woman } & 32 & 27.165 & 0.776 & 29.039 & 0.778 & $\mathbf{3 0 . 7 2 4}$ & $\mathbf{0 . 8 0 8}$ \\
& 64 & 30.106 & 0.831 & 32.682 & 0.858 & $\mathbf{3 4 . 2 9 7}$ & $\mathbf{0 . 8 7 2}$ \\
& 128 & 31.912 & 0.892 & 34.986 & 0.905 & $\mathbf{3 6 . 6 8 2}$ & $\mathbf{0 . 9 2 1}$ \\
& 256 & 34.550 & 0.934 & 37.128 & 0.933 & $\mathbf{3 8 . 8 8 0}$ & $\mathbf{0 . 9 5 0}$ \\
\hline \multirow{4}{*}{ Flowersyyyyyyyy} & 32 & 26.403 & 0.750 & 28.756 & 0.776 & $\mathbf{3 0 . 0 1 7}$ & $\mathbf{0 . 7 9 4}$ \\
& 64 & 27.454 & 0.813 & 31.605 & 0.833 & $\mathbf{3 2 . 1 2 4}$ & $\mathbf{0 . 8 7 4}$ \\
& 128 & 29.963 & 0.853 & 33.527 & 0.902 & $\mathbf{3 5 . 4 0 5}$ & $\mathbf{0 . 9 1 1}$ \\
& 256 & 32.240 & 0.897 & 35.483 & 0.927 & $\mathbf{3 7 . 4 4 6}$ & $\mathbf{0 . 9 3 7}$ \\
\hline \multirow{4}{*}{ House } & 32 & 25.601 & 0.795 & 29.575 & 0.829 & $\mathbf{3 2 . 3 1 0}$ & $\mathbf{0 . 8 7 3}$ \\
& 64 & 27.927 & 0.873 & 31.753 & 0.881 & $\mathbf{3 4 . 5 1 8}$ & $\mathbf{0 . 9 1 6}$ \\
& 128 & 30.454 & 0.916 & 34.162 & 0.924 & $\mathbf{3 5 . 9 4 9}$ & $\mathbf{0 . 9 3 4}$ \\
& 256 & 31.952 & 0.938 & 36.287 & 0.947 & $\mathbf{3 8 . 0 5 9}$ & $\mathbf{0 . 9 5 7}$ \\
\hline \hline
\end{tabular}

[5] R. Gentile, J. Allebach, and E. Walowit. Quantization of color images based on uniform color spaces. Journal of Imaging Technology, 16(1):11-21, 1990.

[6] R. Gentile, E. Walowit, and J. Allebach. Quantization and multilevel halftoning of color images for near-original image quality. Journal of the Optical Society of America A, 7(6):1019-1026, 1990.

[7] M. Gervautz and W. Purgathofer. A simple method for color quantization: Octree quantization. In Graphics Gems, pages 287-293. Academic Press Professional, Inc., San Diego, CA, USA, 1990.

[8] P. Heckbert. Color image quantization for frame buffer display. In Proceedings of SIGGRAPH, volume 16, pages 297-307, 1982.

[9] J. Jarvis, C. Judice, and W. Ninke. A survey of techniques for the display of continuous tone pictures on bilevel displays. Computer Graphics and Image Processing, 5(1):1340, 1976.

[10] G. Joy and Z. Xiang. Center-cut for color-image quantization. The Visual Computer, 10(1):62-66, 1993.

[11] L. Kaplan. Extended fractal analysis for texture classification and segmentation. IEEE Transactions on Image Process, 8(11):1572-1585, 1999.

[12] Y.W. Lim and S.U. Lee. On the color image segmentation algorithm based on the thresholding and the fuzzy c-means techniques. Pattern Recognition, 23:935-952, 1990.

[13] Y. Linde, A. Buzo, and R. M. Gray. An algorithm for vector quantizer desing. IEEE Transactions on Communications, 28(1):84-95, 1980.

[14] Y. Liu and Y. Li. Image feature extraction and segmentation using fractal dimension. In Proceedings of International Conference on Information, Communications and Signal Processing, pages 975-979, 1997.

[15] S. P. Lloyd. Least squares quantization in pcm. IEEE Transactions on Information Theory, 28:129-137, 1982.

[16] M. Mahy, L. Van Eycken, and A. Oosterlinck. Evaluation of uniform color spaces developed after the adoption of CIELAB and CIELUV. Journal of Color Research and Application, 19:105-121, 1994.

[17] H. Matsumoto and K. Sasazaki. Color image compression with vector quantization. In Proceedings of IEEE Conference on Soft Computing in Industrial Applications, pages 84-88, 2008.
[18] S. Novianto, Y. Suzuki, and J. Maeda. Near optimum estimation of local fractal dimension for image segmentation. Pattern Recognition Letters, 24(1):365-374, 2003.

[19] M. Orchard and C. Bouman. Color quantization of images. IEEE Transactions on Signal Processing, 39(12):26772690, 1991.

[20] N. Otsu. A threshold selection method from gray-level histograms. IEEE Transactions on Systems, Man, and Cybernetics, 9(1):62-66, 1979.

[21] D. Ozdemir and L. Akarun. Fuzzy algorithms for combined quantization and dithering. IEEE Transactions on Image Processing, 10:923-931, 2001.

[22] A. Pentland. Fractal-based description of natural scenes. IEEE Transactions on Pattern Analysis and Machine Intelligence, 6:661-674, 1984.

[23] A. Pentland. Shading into texture. Artificial Intelligence, 29:147-170, 1986.

[24] H. Potlapalli and R. Luo. Fractal-based classification of natural textures. IEEE Transactions on Industrial Electronics, 45(1):142-150, 1998.

[25] A. Robertson. The cie 1976 color-difference formulae. Color Research and Application, 2:7-11, 1977.

[26] X. Rui, C. Chang, and T. Srikanthan. On the initialization and training methods for kohonen self-organizing feature maps in color image quantization. In Proceedings of the $1^{\text {st }}$ IEEE international workshop on electronic design, test and applications, pages 321-325, 2002.

[27] N. Sarkar and B. Chaudhuri. An efficient approach to estimate fractal dimension of textural images. Pattern Recognition, 25(9):1035-1041, 1992.

[28] N. Sarkar and B. Chaudhuri. An efficient differential boxcounting approach to compute fractal dimension of image. IEEE Transactions on Systems, Man and Cybernetics, 24(1):115-120, 1994.

[29] P. Scheunders and S. De Backer. oint quantization and error diffusion of color images using competitive learning. In International Conference on Image Processing, pages 811814, 1997.

[30] P. Stucki. MECCA - A multiple-error correcting computation algorithm for bilevel image hardcopy reproduction. Technical Report RZ1060, IBM Research Laboratory, Zurich, Switzerland, 1981 
Communications on Applied Electronics (CAE) - ISSN : 2394 - 4714

Foundation of Computer Science FCS, New York, USA

Volume 3 - No. 7, December 2015 - www.caeaccess.org

[31] M. Swain and D. Ballard. Color indexing. International journal of computer vision, 7(1):11-32, 1991.

[32] E. van den Broek, T. Kok, T. Schouten, and L. Vuurpijl. Human-centered content-based image retrieval. In Proceedings of SPIE, volume 6806, page 54, 2008.

[33] Z. Wang, A. Bovik, H. Sheikh, and E. Simoncelli. Image quality assessment: from error visibility to structural similarity. IEEE Transactions on Image Processing, 13(4):600612, 2004.

[34] X. Wu. Efficient statistical computations for optimal color quantization. In Graphics Gems II, pages 126-133. New York: Academic, James Arvo edition, 1991.

[35] C. Yang and W. Tsai. Color image compression using quantization, thresholding, and edge detection techniques all based on the moment-preserving principle. Pattern Recognition Letters, 19:205-215, 1998.

[36] G. Zorpette. Fractals: not just another pretty picture. IEEE Spectrum, 25(10):29-31, 1988. 